Acknowledgements: Medical writing support was provided by Caroline Spencer and $\mathrm{Dr}$ Sue Chambers (Rx Communications, Mold, UK), funded by Fresenius Kabi SwissBioSim.

Disclosure of Interests: Christian Schwabe: None declared, Chris Wynne: None declared, Andras Illes Employee of: Fresenius Kabi SwissBioSim, Martin Ullmann Employee of: Fresenius Kabi SwissBioSim, Emmanuelle Vincent Employee of: Fresenius Kabi SwissBioSim, Vishal Ghori Employee of: Fresenius Kabi SwissBioSim, Corinne Petit-Frere Employee of: Biosimilars, Fresenius Kabi SwissBioSim, Anne-Sophie Racault Employee of: Fresenius Kabi SwissBioSim, Isabelle Morin Employee of: Former employee of Fresenius Kabi SwissBioSim DOI: 10.1136/annrheumdis-2021-eular.74

\section{AB0196 SURVIVAL ANALYSIS OF TIME TO FIRST ADVERSE DRUG REACTION AND DRUG SURVIVAL IN RHEUMATOID ARTHRITIS PATIENTS TREATED WITH ADALIMUMAB AND ETANERCEPT}

M. Nguyen ${ }^{1}$, K. Velthuis ${ }^{1}$, J. Scholl ${ }^{1}$, J. Jansen ${ }^{1}$, L. Kosse ${ }^{1}$, P. Ten Klooster ${ }^{2,3}$, N. Jessurun ${ }^{1}, \mathrm{H}$. Vonkeman ${ }^{3,4} .{ }^{1}$ Netherlands Pharmacovigilance Centre Lareb, Research Department, 's-Hertogenbosch, Netherlands; ${ }^{2}$ Transparency in Healthcare B.V., Hengelo, Netherlands; ${ }^{3}$ University of Twente, Department of Psychology, Health \& Technology, Enschede, Netherlands; ${ }^{4}$ Medisch Spectrum Twente, Department of Rheumatology and Clinical Immunology, Enschede, Netherlands

Background: Treatment of rheumatoid arthritis (RA) with biologic disease-modifying antirheumatic drugs (bDMARDs) has been common practice in the last two decades. However, differences in patients experiencing adverse drug reactions (ADRs) between individual bDMARDs, such as adalimumab (ADA) and etanercept (ETN), during first time treatment has not been studied yet in real-world settings. Objectives: To compare proportions of RA patients experiencing ADRs as well as survival to first ADR and drug survival during treatment with ADA and ETN. Methods: Retrospective single centre cohort study including adult patients with RA, treated with either ADA or ETN between 1 January 2003 and 30 April 2020. The proportions of patients experiencing an ADR were compared by assessing the percentage of patients, treated with either ADA or ETN, experiencing at least one ADR during their first time treatment. Survival to first ADR and drug survival were assessed by calculating time between start of treatment and first ADR and start of treatment and discontinuation of treatment respectively. Stop and restart of treatment within 90 days was considered as continuous use. Differences in proportions were statistically tested using Fisher's Exact Test. Differences in drug survival between ADA and ETN were tested by Kaplan-Meier analysis and Log Rank tests. Results: A total of 422 patients were included in this study (ADA 259, ETN 163). For 93 patients $(21.2 \%)$ an ADR was registered during first time treatment. The proportion of patients experiencing at least one ADR during their first time treatment was $22.7 \%$ for ADA and $20.2 \%$ for ETN ( $p=0.628)$. Survival time to first ADR did not differ significantly between ADA and ETN (median survival ADA 10.34 years (95\% $\mathrm{Cl}$ [7.62-13.06], median survival ETN not reached, $p=0.109$, figure $1 \mathrm{~A})$. Median drug survival was 1.75 years for ADA $(95 \mathrm{Cl}$ [1.38-2.11]) and 2.68 years for ETN $(95 \% \mathrm{Cl}$ [1.73-3.64]). Drug survival differed significantly $(p<0.001$, figure $1 \mathrm{~B})$.
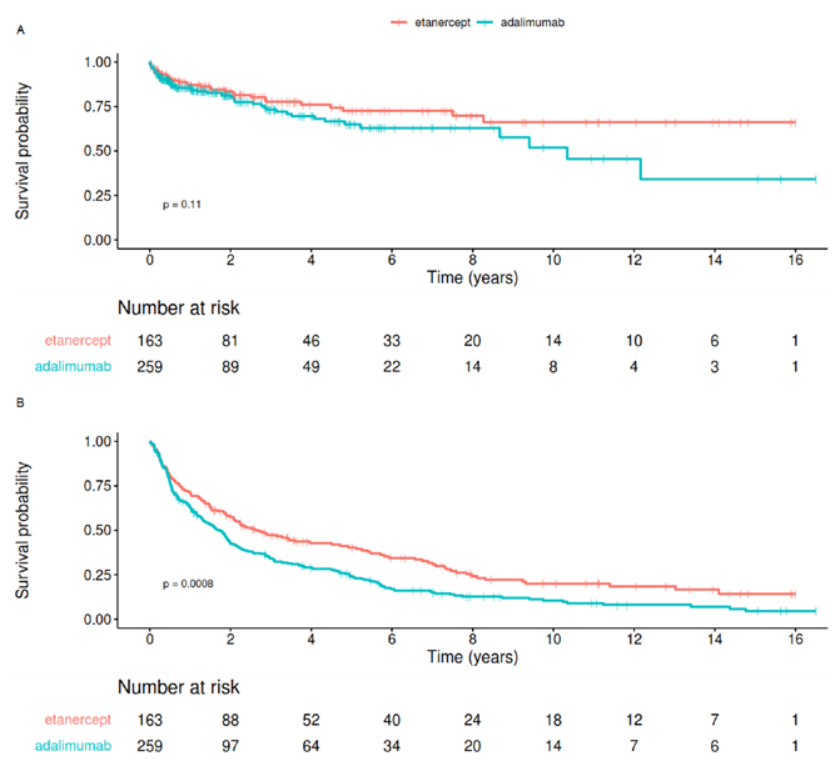

Figure 1. Kaplan-Meier survival curves for adalimumab and etanercept with (a) survival to first ADR and (b) drug survival.
Conclusion: Neither the proportion of patients experiencing ADRs nor survival to first ADR during first time treatment with ADA and ETN differed significantly. Drug survival of first time drug treatment of ADA was significantly lower compared to drug survival of first time drug treatment of ETN.

Disclosure of Interests: My Nguyen: None declared, Kimberly Velthuis: None declared, Joep Scholl: None declared, Jurriaan Jansen: None declared, Leanne Kosse: None declared, Peter ten Klooster: None declared, Naomi Jessurun: None declared, Harald Vonkeman Consultant of: BMS, Celgene, Celltrion, Galapagos, Gilead, Janssen-Cilag, Lilly, Novartis, Pfizer, Sanofi-Genzyme, Grant/ research support from: Abbvie

DOI: 10.1136/annrheumdis-2021-eular.179

\begin{tabular}{|l|}
\hline AB0197 \\
WFFICACY AND SAFETY OF HLX01 COMBINED \\
WITH METHOTREXATE IN CHINESE PATIENTS WITH \\
MODERATELY TO SEVERELY ACTIVE RHEUMATOID \\
ARTHRITIS WHO HAD INADEQUATE RESPONSES \\
TO METHOTREXATE: RESULTS OF A RANDOMISED, \\
DOUBLE-BLIND, PLACEBO-CONTROLLED PHASE 3 \\
STUDY
\end{tabular}

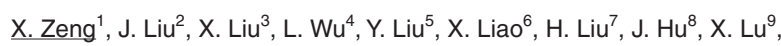
L. Chen ${ }^{10}$, J. Xu ${ }^{11}$, Z. Jiang ${ }^{12}$, F. Lu ${ }^{13}$, H. Wu ${ }^{14}$, L. Sun ${ }^{15}$, M. Wang ${ }^{16}, X . \mathrm{Yu}^{17}$, Q. Wang ${ }^{18} .^{1}$ Peking Union Medical College Hospital, Department of Rheumatology, Beijing, China; '2 Jiujiang NO.1 People's Hospital, Department of Rheumatology, Jiujiang, China; ${ }^{3}$ First Hospital of Shanxi Medical University, Department of Rheumatology, Taiyuan, China; ${ }^{4}$ People's Hospital of Xinjiang Uygur Autonomous Region, Department of Rheumatology, Urumchi, China; ${ }^{5}$ West China Hospital of Sichuan University, Department of Rheumatology, Chengdu, China; ${ }^{6}$ Chenzhou First People's Hospital, Department of Nephrology Chenzhou, China; ${ }^{7}$ Qilu Hospital of Shandong University, Department of Rheumatology, Ji'nan, China; ${ }^{8}$ Jiangxi Pingxiang People's Hospital, Department of Rheumatology, Pingxiang, China; ${ }^{9}$ China-Japan Friendship Hospital, Department of Rheumatology, Beijing, China; ${ }^{10}$ The First Affiliated Hospital of Bengbu Medical College, Department of Rheumatology, Bengbu, China; ${ }^{11}$ First Affiliated Hospital of Kunming Medical University, Department of Rheumatology, Kunming, China; ${ }^{12}$ The First Hospital of Jilin University, Department of Rheumatology, Changchun, China; ${ }^{13}$ The First Affiliated Hospital of Baotou Medical College, Inner Mongolia University of Science and Technology, Department of Rheumatology, Baotou, China; ${ }^{14}$ The Second Affiliated Hospital Zhejiang University School of Medicine, Department of Rheumatology, Hangzhou, China; ${ }^{15}$ Nanjing Drum Tower Hospital, Department of Rheumatology, Nanjing, China; ${ }^{16}$ Zhongda Hospital Southeast University, Department of Rheumatology, Nanjing, China: ${ }^{17}$ Cangzhou Hospital of Integrated TCM-WM - Hebei, Department of Rheumatology, Cangzhou, China ${ }^{18}$ Shanghai Henlius Biotech, Inc., Global Product Development, Shanghai, China

Background: Rituximab is an effective therapy for rheumatoid arthritis (RA) patients with inadequate responses to methotrexate (MTX $)^{1,2}$. However, it has not been registered or approved in China for the treatment of RA by far. HLX01, an approved rituximab biosimilar (demonstrated in Chinese patients with diffuse large B-cell lymphoma) ${ }^{3}$, is thus evaluated in this study for the benefits of Chinese RA patients.

Objectives: This study aimed to evaluate the efficacy and safety of HLX01 plus MTX versus placebo plus MTX in Chinese patients with active RA who had inadequate responses to MTX.

Methods: This was a randomised, double-blind, placebo-controlled phase 3 study conducted in China (NCT03522415). Eligible patients were randomised 2:1 to receive intravenous infusion of $2 \times 1000 \mathrm{mg} \mathrm{HLX01}$ or placebo on day 1 and day 15 . Patients with inadequate responses at week 16 and 20 were allowed to receive rescue treatments. Patients were retreated with or switched to receive (if initially assigned to placebo) $2 \times 1000 \mathrm{mg}$ rituximab at the first day of week 24 and 26. The primary endpoint of this study was the American College of Rheumatology criteria (ACR) 20 response at week 24 . Secondary efficacy endpoints were evaluated at week $12,24,36$ and 48 . The safety, pharmacokinetics, pharmacodynamics and immunogenicity of HLX01 were observed and analyzed throughout the study.

Results: Between May 28, 2018 and Sep 11, 2020, a total of 275 patients (ITT set) were randomised and 263 patients without major protocol deviations were included in per-protocol set (PPS). At week 24, HLX01 showed statistically superior efficacy ( $p<0.001$ ) to placebo (ACR20: $60.7 \%$ vs $35.9 \%$ in ITT set, $60.3 \%$ vs $37.1 \%$ in PPS). Secondary efficacy endpoints were also significantly improved in HLX01 group compared with placebo (Table 1). The overall incidence of serious treatment emergent adverse events (TEAEs), adverse drug reactions (ADRs), and TEAEs leading to drug discontinuation were similar among treatment groups, with the most common TEAE been upper respiratory tract infection before $(18.1 \%$ vs $18.5 \%)$ or after $(13.0 \%$ vs $12.3 \%$ ) week 24 . Serum concentrations, immunogenicity and pharmacodynamics were similar between $\mathrm{HLX} 01$ and placebo groups. 
Table 1. Results of secondary efficacy endpoints at week 12, 24, 36 and 48 in ITT set.

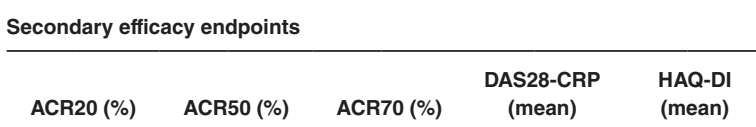

Duration HLX01 Placebo HLX01 Placebo HLX01 Placebo HLX01 Placebo HLX01 Placebo

\begin{tabular}{lllllllllll}
\hline Baseline & & & & & & & 5.49 & 5.43 & 1.40 & 1.45 \\
Week 12 & 48.1 & 32.6 & 21.9 & 10.9 & 4.4 & 5.4 & 3.89 & 4.47 & 1.02 & 1.22 \\
Week 24 & 60.7 & 35.9 & 36.6 & 18.5 & 15.3 & 12.0 & 3.39 & 4.37 & 0.87 & 1.22 \\
Week 36 & 60.1 & 48.9 & 46.4 & 31.5 & 32.2 & 17.4 & 2.88 & 3.51 & 0.71 & 0.97 \\
Week 48 & 73.8 & 62.0 & 55.2 & 40.2 & 39.9 & 27.2 & 2.82 & 3.51 & 0.72 & 1.03
\end{tabular}

Conclusion: Comparing with placebo plus MTX, HLX01 plus MTX showed significantly improved clinical outcomes and comparable safety profiles in Chinese patients with moderately to severely active RA who had inadequate responses to MTX, demonstrating HLX01 in combination with MTX as a well-tolerated, safe and efficient treatment option.

\section{REFERENCES:}

[1] Emery P, Deodhar A, Rigby WF, et al. Efficacy and safety of different doses and retreatment of rituximab: a randomised, placebo-controlled trial in patients who are biological naive with active rheumatoid arthritis and an inadequate response to methotrexate (Study Evaluating Rituximab's Efficacy in MTX iNadequate rEsponders (SERENE)). Ann Rheum Dis. Sep 2010;69(9):1629-35. doi:10.1136/ard.2009.119933.

[2] Rubbert-Roth A, Tak PP, Zerbini C, et al. Efficacy and safety of various repeat treatment dosing regimens of rituximab in patients with active rheumatoid arthritis: results of a Phase III randomized study (MIRROR). Rheumatology (Oxford). Sep 2010;49(9):1683-93. doi:10.1093/rheumatology/ keq116.

[3] Shi Y, Song Y, Qin Y, et al. A phase 3 study of rituximab biosimilar HLX01 in patients with diffuse large B-cell lymphoma. $J$ Hematol Oncol. Apr 16 2020;13(1):38. doi:10.1186/s13045-020-00871-9.

Acknowledgements: The authors would like to thank participants in this study and their families. They would also like to acknowledge other investigators and staff at all clinical sites and the members of the Independent Data Monitoring Committee.

Disclosure of Interests: None declared

DOI: 10.1136/annrheumdis-2021-eular.282

\section{AB0198 $\quad$ EFFICACY AND SAFETY AFTER TRANSITION FROM REFERENCE ADALIMUMAB TO CT-P17 (ADALIMUMAB BIOSIMILAR: $100 \mathrm{MG} / \mathrm{ML}$ ) IN COMPARISON WITH THE MAINTAINED TREATMENT (CT-P17 OR REFERENCE ADALIMUMAB) IN PATIENTS WITH MODERATE-TO- SEVERE ACTIVE RHEUMATOID ARTHRITIS: 1-YEAR RESULT}

D. Furst ${ }^{1}$, E. Keystone ${ }^{2}$, J. Kay ${ }^{3}$, J. Jaworski ${ }^{4}$, R. Wojciechowski ${ }^{5}$, P. Wiland ${ }^{6}$, A. Dudek ${ }^{7}$, M. Krogulec ${ }^{8}$, S. Jeka ${ }^{9}$, A. Zielinska ${ }^{10}$, J. Trefler $^{11}$, K. BartnickaMasłowska $^{12}$, M. Krajewska-Wlodarczyk ${ }^{13}$, P. Klimiuk ${ }^{14}$, S. J. Lee ${ }^{15}$, S. H. Kim ${ }^{16}$, Y. Bae ${ }^{16}$, G. Yang ${ }^{16}$, J. Yoo ${ }^{16}$, T. Kim $^{17} .{ }^{1}$ University of California, Rheumatology, Los Angeles, United States of America; ${ }^{2}$ University of Toronto, Medicine, Toronto, Canada; ${ }^{3}$ University of Massachusetts Medical School, Medicine and Population \& Quantitative Health Sciences, Worcester, United States of America; ${ }^{4}$ Reumatika Centrum Reumatologii, Reumatika Centrum Reumatologii, Warsaw, Poland; ${ }^{5}$ University Hospital No 2 in Bydgoszcz, Rheumatology and Connective Tissue Diseases, Bydgoszcz, Poland; ${ }^{6}$ Wroclaw Medical University, Rheumatology and Internal Diseases, Wrocław, Poland; ${ }^{7}$ Centrum Medyczne AMED, Reumatologia, Warsaw, Poland; ${ }^{8}$ Reumatology Clinic NZOZ Lecznica MAK-MED, Rheumatology, Nadarzyn, Poland; ${ }^{9}$ Nasz Lekarz Przychodnie Medyczne, Not applicable, Toruń, Poland; ${ }^{10}$ Medycyna Kliniczna, Not applicable, Warszawa, Poland; ${ }^{11}$ Reuma Centrum, Rheumatology, Warsaw, Poland;

${ }^{12}$ Centrum Medyczne AMED, Oddział w Łodzi, Lodz, Poland; ${ }^{13}$ University of Warmia and Mazury in Olsztyn, Rheumatology, Olsztyn, Poland; ${ }^{14}$ Medical University of Bialystok, Rheumatology and Internal Diseases, Bialystok, Poland; ${ }^{15}$ Celltrion, Inc, Clinical Development Division, Incheon, Korea, Rep. of (South Korea); ${ }^{16}$ Celltrion, Inc, Clinical Planning, Incheon, Korea, Rep. of (South Korea); ${ }^{17}$ Celltrion, Inc, Biometrics, Incheon, Korea, Rep. of (South Korea)

Background: Therapeutic equivalence of CT-P17 to reference adalimumab (ref-adalimumab) has been shown in patients with moderate-to-severe active rheumatoid arthritis (RA) through primary 24-week results [1]. Here, efficacy, pharmacokinetics (PK), safety and immunogenicity results up to 52-week, including transition data from ref-adalimumab to CT-P17 are presented.
Objectives: To evaluate efficacy, PK, safety and immunogenicity when switched from ref-adalimumab to CT-P17 compared to maintaining CT-P17 or ref-adalimumab.

Methods: In this study, 648 moderate-to-severe active RA patients despite methotrexate treatment were randomized $(1: 1)$ to either CT-P17 or ref-adalimumab and treated with doses of $40 \mathrm{mg}$ every 2 weeks up to Week 24. Prior to dosing at Week 26, 608 patients were randomized again to either maintaining their treatments or being switched from ref-adalimumab to CT-P17. Efficacy, PK, safety, and immunogenicity were assessed up to Week 52 .

Results: After the second randomization, 303 patients continued with CT-P17, 153 patients continued with ref-adalimumab and 151 patients switched from ref-adalimumab to CT-P17 treatments, up to Week 48. Demographics and baseline characteristics were similar among the 3 groups. Sustained and comparable efficacy in terms of ACR20/50/70 response rates was achieved not only in the maintenance groups (CT-P17 or ref-adalimumab) but also in the switched from ref-adalimumab to CT-P17 group up to Week 52 (Figure 1)

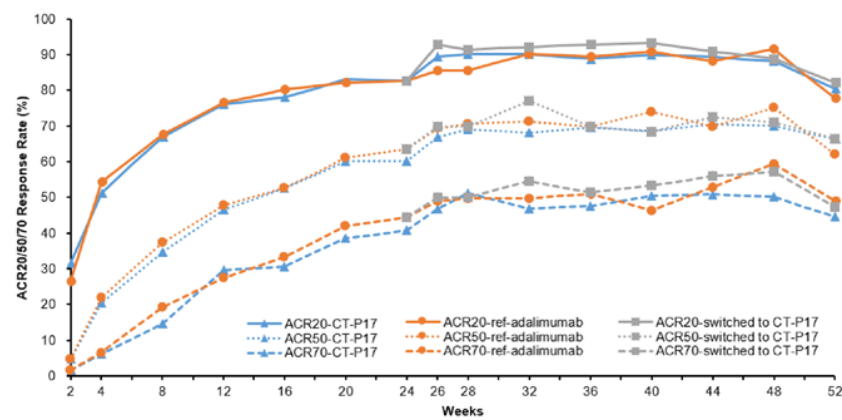

Figure 1. ACR 20/50/70 Response Rates up to 1 YearAbbreviation: ref-adalimumab, reference adalimumab.Note. There were patients who could not visit the study site due to COVID-19 pandemic and were counted as nonresponder for ACR response at Week 52.

In terms of PK, mean trough serum concentration (Ctrough) were maintained after Week 24 in all 3 groups. The observed mean Ctrough were within the reported therapeutic ranges of ref-adalimumab trough levels in RA patients (5-8 $\mu \mathrm{g} / \mathrm{mL})$.

The safety profile after transition was comparable among the 3 groups (Table 1). The most common treatment-emergent adverse events (TEAEs) was neutropenia. Similar proportions of patients in all 3 groups experienced at least 1 TEAE: injection site reactions, hypersensitivity/allergic reactions and infections. One malignancy (basal cell carcinoma; unrelated) was reported in the ref-adalimumab maintenance group. Safety data accumulated over 1 year also showed comparable results among the 3 groups. Anti-drug antibody (ADA) and neutralizing antibody (NAb) results were similar among the 3 groups. At Week 52 , the proportions of patients who had ADA/NAbs were $28.4 \% / 24.8 \%$ patients in CT-P17 maintenance, $27.0 \% / 24.3 \%$ patients in ref-adalimumab maintenance and $28.3 \% / 26.3 \%$ patients in switched to CT-P17 groups.

Conclusion: Single transition from ref-adalimumab to CT-P17 was efficacious and safe without increase in immunogenicity. Also, efficacy, PK, safety and immunogenicity profiles were comparable between CT-P17 and ref-adalimumab up to Week 52.

REFERENCES:

[1] J Kay et al, 2020. Poster Presented at ACR Convergence 2020.

Table 1. Overview of TEAEs from Weeks 26 to 52 (Safety Population second random subset)

\begin{tabular}{|c|c|c|c|}
\hline \multirow[b]{2}{*}{ Patients, n (\%) } & \multicolumn{3}{|c|}{ Second Randomization } \\
\hline & $\begin{array}{c}\text { CT-P17 } \\
\text { Maintenance } \\
(\mathrm{N}=303)\end{array}$ & $\begin{array}{c}\text { Ref-ada } \\
\text { Maintenance } \\
(\mathrm{N}=152)\end{array}$ & $\begin{array}{l}\text { Switched to } \\
\text { CT-P17 (N=152) }\end{array}$ \\
\hline$\geq 1 \mathrm{TEAE}$ & $121(39.9)$ & $69(45.4)$ & $73(48.0)$ \\
\hline$\geq 1 \mathrm{TESAE}$ & $6(2.0)$ & $3(2.0)$ & $5(3.3)$ \\
\hline $\begin{array}{l}\geq 1 \text { TEAE leading to study drug } \\
\text { discontinuation }\end{array}$ & $3(1.0)$ & $2(1.3)$ & $5(3.3)$ \\
\hline $\begin{array}{l}\geq 1 \text { TEAE classified as hypersensitivity/ } \\
\quad \text { allergic reactions }\end{array}$ & $2(0.7)$ & $1(0.7)$ & $0(0)$ \\
\hline $\begin{array}{l}\geq 1 \text { TEAE classified as injection site } \\
\text { reactions }\end{array}$ & $1(0.3)$ & $4(2.6)$ & $1(0.7)$ \\
\hline$\geq 1$ TEAE classified as infection & $54(17.8)$ & $41(27.0)$ & $28(18.4)$ \\
\hline$\geq 1$ TEAE classified as malignancy & $0(0)$ & $1(0.7)$ & $0(0)$ \\
\hline
\end{tabular}

Abbreviations: Ref-ada, reference adalimumab; TEAE, treatment-emergent adverse event TESAE, treatment-emergent serious adverse event. 